\title{
Alfvén wave dissipation and topological properties of 3D coronal force-free magnetic fields
}

\author{
F. Malara, P. Veltri, and M. F. De Franceschis
}

Dipartimento di Fisica, Università della Calabria, via P. Bucci, 87036 Rende (CS), Italy

e-mail: malara@fis.unical.it

Received 27 September 2006 / Accepted 7 February 2007

\section{ABSTRACT}

\begin{abstract}
We study the propagation and dissipation of Alfvénic perturbation in a 3D equilibrium structure within a WKB model. We assume small amplitude and small wavelength of the perturbation. The generation of small scales in the perturbation is related to the property that nearby magnetic lines move apart from each other locally. This property is quantified by means of the Kolmogorov entropy $H$ of magnetic lines. We numerically calculate the distribution of $H$ for a 3D complex force-free equilibrium, which models the magnetic field above a quiet-Sun region, both for nonvanishing current and for a potential field. It is found that $H$ decreases slightly with the altitude due to the decreasing complexity of the field, but it is relatively uniform except for the presence of sharp peaks, where $H$ reaches much higher values than the average. These locations are those where Alfvén waves are preferentially dissipated. By analyzing the magnetic topology at these locations, we find that they correspond to separator lines which are intersections of separatrix surfaces. Then, in a high-Reynolds number plasma, such as in the solar corona, heating due to Alfvén wave dissipation takes place mainly at magnetic separatrices.
\end{abstract}

Key words. magnetohydrodynamics (MHD) - waves - Sun: magnetic fieds

\section{Introduction}

Dissipation of Alfvénic fluctuations is one of the mechanisms invoked to explain the nonradiative heating of the solar corona. While the amount of energy flux carried by these fluctuations is probably sufficient for explaining the observed temperature of the coronal plasma, the main theoretical problem is how to efficiently dissipate this energy, due to the very low value of dissipative coefficients (resistivity and viscosity). When propagating in a highly nonuniform medium like the coronal plasma, Alfvén waves couple with the spatial inhomogeneities of both the density and magnetic field. The result is that the wave energy can be moved to increasingly smaller scales, eventually reaching dissipative scales where it is converted into heat. In this framework, it is important to evaluate whether in a given equilibrium configuration such dynamical mechanisms of small-scale generation are efficient enough to dissipate a perturbation before it leaves the corona.

In a 2D inhomogeneous background, where the Alfvén velocity $c_{\mathrm{A}}$ varies in directions perpendicular to the magnetic field, two mechanisms have been studied in detail: (1) phase-mixing (Heyvaerts \& Priest 1983), in which differences in phase velocity at different locations progressively bend wavefronts; and (2) resonant absorption which concentrates the wave energy in a narrow layer where the wave frequency locally matches a characteristic frequency (Alfvén or cusp). These processes have been studied both by investigating normal modes of the inhomogeneous structure (Kappraff \& Tataronis 1977; Mok \& Einaudi 1985; Steinolfson 1985; Davila 1987, 1991; Califano et al. 1990, 1992; Hollweg 1987a,b) and by considering the time evolution of an initial disturbance (Lee \& Roberts 1986; Malara et al. 1992, 1996). Effects of density stratification and magnetic line divergence (Ruderman et al. 1998), as well as nonlinear coupling with compressive modes (Nakariakov et al. 1997, 1998), have also been considered.

A full description of wave propagation in a 3D magnetic field represents a formidable task, since different wave modes (Alfvén and magnetosonic) are coupled among them and with the inhomogeneity of the background structure. A possible approach is represented by direct numerical MHD simulations. However, for a 3D configuration, the limited number of numerical degrees of freedom makes the high Reynolds/Lundquist numbers that are typical of the coronal plasma inaccessible.

An alternative approach to such a problem in 3D equilibria was first proposed by Similon \& Sudan (1989). In a 3D equilibrium configuration, there can be regions where magnetic lines show a chaotic behavior: nearby magnetic lines can exponentially move apart with increasing distance along the lines (Zimbardo et al. 1984, 1995). Then, Alfvénic disturbances that locally follow magnetic lines are stretched exponentially in time, leading to a fast formation of small scales. As a consequence, the typical dissipation time $t_{\mathrm{d}}$ of fluctuations follows the scaling law:

$t_{\mathrm{d}} \propto \log S$

where $S$ is the relevant viscous and/or resistive Reynolds number. Thus, $t_{\mathrm{d}}$ remains relatively small even for high values of $S$, as in the coronal plasma. This mechanism has been investigated by Petkaki et al. (1998) and by Malara et al. (2000), who built up a model describing propagation and dissipation of Alfvénic wave packets in $3 \mathrm{D}$ magnetic structures, both in the incompressible and in the compressible low- $\beta$ case (Malara et al. 2003). These authors show that the scaling law (1) is recovered in regions of chaotic magnetic lines, while a slower dissipation related to phase-mixing takes place in regions where the topology of magnetic lines is quasi-2D. Recently (Malara et al. 2005, Paper I), we have used the above model to study Alfvén wave 
dissipation in a 3D force-free magnetic equilibrium with a complex topology, which represents a model for the coronal magnetic field above a quiet-Sun region. The effect of a partial reflection at the coronal base was included, in order to evaluate a balance between wave dissipation and energy leakage from the corona.

The aim of the present paper is to study in more detail how the topology of magnetic lines determines the generation of small scales in Alfvénic perturbations. This property will be characterized in a quantitative way by using the Kolmogorov entropy of magnetic lines. This method is applied to the force-free magnetic equilibrium introduced by Nakagawa \& Raadu (1972) and considered in Paper I. In particular, we identify the topology of the regions where the rate of small-scale formation is the highest. In turn, this allows us to determine the magnetic regions where the heat deposition due to Alfvén wave dissipation is particularly large.

\section{Alfvén waves in an inhomogeneous magnetic field}

The coronal plasma is characterized by a low value of the plasma $\beta$ (the gas pressure to magnetic pressure ratio). Thus, the usual representation of large-scale phenomena is the one given by the cold plasma MHD equations, in which pressure and gravity terms are neglected with respect to the magnetic force in the momentum equation. These equations are written in the following dimensionless form:

$\frac{\partial \rho}{\partial t}+v_{j} \frac{\partial \rho}{\partial x_{j}}+\rho \frac{\partial v_{j}}{\partial x_{j}}=0$

$$
\begin{aligned}
\rho\left[\frac{\partial v_{i}}{\partial t}+v_{j} \frac{\partial v_{i}}{\partial x_{j}}\right]= & -\frac{\partial}{\partial x_{i}}\left(\frac{B_{j} B_{j}}{8 \pi}\right)+\frac{1}{4 \pi} B_{j} \frac{\partial B_{i}}{\partial x_{j}} \\
& +\eta_{N} \frac{\partial^{2} v_{i}}{\partial x_{j} \partial x_{j}}+\left(\zeta_{N}+\frac{\eta_{N}}{3}\right) \frac{\partial}{\partial x_{i}} \frac{\partial v_{j}}{\partial x_{j}}
\end{aligned}
$$

$\frac{\partial B_{i}}{\partial t}+v_{j} \frac{\partial B_{i}}{\partial x_{j}}=B_{j} \frac{\partial v_{i}}{\partial x_{j}}-B_{i} \frac{\partial v_{j}}{\partial x_{j}}+\lambda_{N} \frac{\partial^{2} B_{i}}{\partial x_{j} \partial x_{j}}$.

Here, lengths have been normalized to a characteristic length $L$, the magnetic field is normalized to a characteristic magnetic field $\hat{B}_{0}$, the density to a characteristic density $\hat{\rho}_{0}$, the velocity to the corresponding Alfvén velocity $\hat{c}_{\mathrm{A} 0}=\hat{B}_{0} /\left(4 \pi \hat{\rho}_{0}\right)^{1 / 2}$, and the time to the Alfvén time $t_{\mathrm{A}}=L / \hat{c}_{\mathrm{A} 0}$. The dimensionless dissipative coefficients are $\eta_{N}=\eta / \hat{\rho}_{0} L \hat{c}_{\mathrm{A} 0}, \zeta_{N}=\zeta / \hat{\rho}_{0} L \hat{c}_{\mathrm{A} 0}$, and $\lambda_{N}=\lambda / L \hat{c}_{\mathrm{A} 0}$, where $\eta$ and $\zeta$ are the viscosity coefficients and $\lambda$ is the magnetic diffusivity, which are assumed to be constant. Summation over dummy indices is hereafter understood.

We consider the propagation and the dissipation of Alfvén waves in a $3 \mathrm{D}$ inhomogeneous magnetic structure, using a method (Malara et al. 2003) that is summarized in the following. Density, velocity, and magnetic field are written in the following form:

$$
\begin{aligned}
\rho(\boldsymbol{x}, t) & =\rho^{(0)}(\boldsymbol{x})+\varepsilon \rho^{(1)}(\boldsymbol{x}, t) \\
\boldsymbol{v}(\boldsymbol{x}, t) & =\boldsymbol{\varepsilon} \boldsymbol{v}^{(1)}(\boldsymbol{x}, t) \\
\boldsymbol{B}(\boldsymbol{x}, t) & =\boldsymbol{B}^{(0)}(\boldsymbol{x})+\boldsymbol{\varepsilon} \boldsymbol{B}^{(1)}(\boldsymbol{x}, t)
\end{aligned}
$$

where $\varepsilon \ll 1$ represents the perturbation amplitude. The upper indices "(0)" and "(1)" denotes quantities relative to the equilibrium and to the perturbation, respectively. We assume that equilibrium quantities $\rho^{(0)}(\boldsymbol{x})$ and $\boldsymbol{B}^{(0)}(\boldsymbol{x})$ vary on a scale denoted by $L_{0}$. The velocity is vanishing at the order $\varepsilon^{0}$ of the expansion, corresponding to a static structure.

Equations (2)-(4) are linearized with respect to the perturbation amplitude, thus obtaining the perturbation evolution equations. Another assumption is that the wavelength $\lambda$ of the perturbation stays much smaller than the scale length $L_{0}$ of the equilibrium structure $\left(\lambda \ll L_{0}\right)$. During the evolution, the wavelength $\lambda$ tends to decrease in time (Petkaki et al. 1998). Thus, the above condition is always fulfilled, provided that it is satisfied at the initial time. This assumption allows a WKB expansion of linearized equations to be performed using wavelength as the expansion parameter. The perturbation is considered as a superposition of different modes, propagating independently from one another. Perturbed quantities are expanded up to the first order in a parameter $\delta \sim \lambda$ :

$f^{(1)}=\mathfrak{R}\left[\sum_{\alpha}\left(f_{0}^{\alpha}+\delta f_{1}^{\alpha}\right) \exp \left(i \frac{S^{\alpha}}{\delta}\right)\right]$

where $f^{(1)}$ represents any perturbation quantity. In this equation " $\alpha$ " indicates the different propagating modes; the quantity $S^{\alpha}(\boldsymbol{x}, t)$ is real and represents the phase of the $\alpha$-th mode; the indices " 0 " and " 1 " indicate $O\left(\delta^{0}\right)$ and $O\left(\delta^{1}\right)$ quantities, respectively. The frequency $\omega^{\alpha}$ and wavevector $\boldsymbol{k}^{\alpha}$ of the $\alpha$-th mode depend both on $\boldsymbol{x}$ and $t$. They are defined by

$\omega^{\alpha}(\boldsymbol{x}, t)=-\frac{1}{\delta} \frac{\partial S^{\alpha}}{\partial t}$

$k_{n}^{\alpha}(\boldsymbol{x}, t)=\frac{1}{\delta} \frac{\partial S^{\alpha}}{\partial x_{n}}$

Finally, dissipative coefficients are supposed to be small. We assume that dissipation coefficients are quantities of order of magnitude $O\left(\delta^{2}\right)$. Then, dissipative coefficients do not affect the wave propagation, but they enter into the equation of energy determining the wave dissipation (Malara et al. 2003). Expanding the linearized MHD equations with respect to the parameter $\delta$, at the lowest order $\delta^{-1}$, the polarization vectors and the dispersion relation of each propagating mode are derived. The dispersion relation of Alfvénic perturbation is

$\omega^{ \pm \mathrm{A}}= \pm c_{\mathrm{A} j}^{(0)} k_{j}$

where $\boldsymbol{c}_{\mathrm{A}}^{(0)}(\boldsymbol{x})=\boldsymbol{B}^{(0)}(\boldsymbol{x}) /\left[\rho^{(0)}(\boldsymbol{x})\right]^{1 / 2}$ is the background Alfvén velocity, while the sign " \pm " identifies waves propagating parallel or antiparallel to the background magnetic field $\boldsymbol{B}^{(0)}$, respectively. Velocity and magnetic field perturbations are polarized perpendicular both to $\boldsymbol{k}$ and to $\boldsymbol{B}^{(0)}$, while the density perturbation is vanishing. As usual, rays are defined as those trajectories in the space-time, along which phase $S^{\alpha}(\boldsymbol{x}, t)$ of the $\alpha$-th mode remains constant. The equation defining the rays of Alfvén waves are derived at the order $\delta^{0}$ of the expansion:

$\frac{\mathrm{d} x_{i}^{ \pm \mathrm{A}}}{\mathrm{d} t}= \pm c_{\mathrm{A}}^{(0)} b_{i}^{(0)}$

where $\boldsymbol{x}=\boldsymbol{x}(t)$ is the position of a point on a wavefront at time $t$, and $\boldsymbol{b}^{(0)}=\boldsymbol{B}^{(0)} / \boldsymbol{B}^{(0)}$ is the unit vector in the direction of the equilibrium magnetic field $\boldsymbol{B}^{(0)}$. This equation indicates that each point on a wavefront of an Alfvénic perturbation propagates along the background magnetic field at the local Alfvén velocity. When $\boldsymbol{c}_{\mathrm{A}}^{(0)}$ is inhomogeneous, different parts of a wavefront propagate at different speeds. Thus, plane wavefronts are bent and distorted, and increasingly smaller scales generate in 
the perturbation. The wavevector $\boldsymbol{k}^{ \pm \mathrm{A}}$ varies along the rays according to the equation

$\frac{\mathrm{d} k_{i}^{ \pm \mathrm{A}}}{\mathrm{d} t}=\mp \frac{\partial\left(c_{\mathrm{A}}^{(0)} b_{n}^{(0)}\right)}{\partial x_{i}} k_{n}^{ \pm \mathrm{A}}$

where $\mathrm{d} / \mathrm{d} t=\partial / \partial t+\left(\mathrm{d} x_{n} / \mathrm{d} t\right)\left(\partial / \partial x_{n}\right)$ indicates the time derivative along the rays.

\section{Small-scale formation and the Kolmogorov entropy of magnetic lines}

The set of Eqs. (11), (12) can be solved exactly in the special case of phase-mixing. Assuming that the Alfvén speed is directed in the $z$ direction and varies along the $x$ direction: $\boldsymbol{c}_{\mathrm{A}}^{(0)}=c_{\mathrm{A}}^{(0)}(x) \boldsymbol{e}_{z}$ (where $\boldsymbol{e}_{z}$ is the unit vector along $z$ ), the solution of Eqs. (11), (12) is

$\boldsymbol{x}(t)=x_{0} \boldsymbol{e}_{x}+y_{0} \boldsymbol{e}_{y}+\left[z_{0} \pm c_{\mathrm{A}}^{(0)}\left(\boldsymbol{x}_{0}\right) t\right] \boldsymbol{e}_{z}$

and

$\boldsymbol{k}^{ \pm \mathrm{A}}(t)=\left(k_{0 x} \mp \frac{\mathrm{d} c_{\mathrm{A}}^{(0)}}{\mathrm{d} x} k_{0 z} t\right) \boldsymbol{e}_{x}+k_{0 y} \boldsymbol{e}_{y}+k_{0 z} \boldsymbol{e}_{z}$

where $\left(k_{0 x}, k_{0 y}, k_{0 z}\right)$ is the initial wavevector at the initial position $\left(x_{0}, y_{0}, z_{0}\right)$. Thus, after a transient, the wavevector $k^{ \pm \mathrm{A}}$ increases proportional to time $t$, which is typical of phase-mixing (Heyvaerts \& Priest 1983). In more complex 3D configurations, the numerical solution of Eqs. (11), (12) (Malara et al. 2000) has shown that there can be regions where the growth of the wavevector $k^{ \pm \mathrm{A}}$ proceeds exponentially in time, which is much faster than in phase-mixing. In such regions magnetic lines are chaotic, i.e., the distance between two nearby lines increases exponentially along the lines (Similon \& Sudan 1989).

The evolution equation for small volume elements of the Alfvénic perturbation can be derived. First, we consider two points within the perturbation: $\boldsymbol{x}(t)$ and $\boldsymbol{x}^{\prime}(t)=\boldsymbol{x}(t)+\delta \boldsymbol{x}(t)$, where $\delta \boldsymbol{x}(t)$ is the infinitesimal vector difference. The evolution equation for $\delta \boldsymbol{x}$ is derived by expanding Eq. (11) to the first order:

$\frac{\mathrm{d} \delta x_{i}}{\mathrm{~d} t}= \pm \frac{\partial c_{\mathrm{A} i}^{(0)}}{\partial x_{n}} \delta x_{n}$

Combining Eqs. (12) and (15), we find

$\frac{\mathrm{d}\left(k_{i} \delta x_{i}\right)}{\mathrm{d} t}=\mp \delta x_{i} \frac{\partial c_{\mathrm{A} n}^{(0)}}{\partial x_{i}} k_{n} \pm k_{i} \frac{\partial c_{\mathrm{A} i}^{(0)}}{\partial x_{n}} \delta x_{n}=0$.

Thus, the quantity $\boldsymbol{k} \cdot \delta \boldsymbol{x}=k \delta x_{\|}$remains constant along the rays (where $\delta x_{\|}$is the component of $\delta \boldsymbol{x}$ parallel to the wavevector $\boldsymbol{k}$ ). If initially $\delta x_{\|}=0$, then $\delta x_{\|}$keeps vanishing all along the ray trajectory: this corresponds to points $\boldsymbol{x}$ and $\boldsymbol{x}^{\prime}$ on the same wave front, and it indicates that the wavevector $\boldsymbol{k}$ remains locally perpendicular to wavefronts along each ray. In contrast, if $\boldsymbol{x}$ and $\boldsymbol{x}^{\prime}$ are located on two different wavefronts so that $\delta x_{\|} \neq 0$, then $\delta x_{\|}$ is proportional to $k^{-1}$. In other words, the distance $\delta x_{\|}$between wavefronts decreases locally with increasing the wavevector $k$.

Another result can be derived from Eq. (15). We consider three non-coplanar vector differences: $\delta \boldsymbol{x}^{A}, \delta \boldsymbol{x}^{B}$, and $\delta \boldsymbol{x}^{C}$ defining an infinitesimal portion of the perturbation, whose volume is $\delta V=|\delta U|$, with

$\delta U=\delta \boldsymbol{x}^{A} \cdot\left(\delta \boldsymbol{x}^{B} \times \delta \boldsymbol{x}^{C}\right)=\varepsilon_{i j k} \delta x_{i}^{A} \delta x_{j}^{B} \delta x_{k}^{C}$ where $\varepsilon_{i j k}$ is the Ricci's tensor. Using the Eq. (15) and the property $\varepsilon_{i j k}=\varepsilon_{k i j}=\varepsilon_{j k i}$, the evolution equation for $\delta U$ can be written in the form

$$
\frac{\mathrm{d} \delta U}{\mathrm{~d} t}= \pm \varepsilon_{i j k} \frac{\partial c_{\mathrm{A} i}^{(0)}}{\partial x_{n}}\left(\delta x_{n}^{A} \delta x_{j}^{B} \delta x_{k}^{C}+\delta x_{k}^{A} \delta x_{n}^{B} \delta x_{j}^{C}+\delta x_{j}^{A} \delta x_{k}^{B} \delta x_{n}^{C}\right),
$$

which can be simplified to

$\frac{\mathrm{d} \delta U}{\mathrm{~d} t}=\left(\nabla \cdot \boldsymbol{c}_{\mathrm{A}}^{(0)}\right) \delta U=-\frac{\delta U}{2 \rho^{(0)}} \boldsymbol{c}_{\mathrm{A}}^{(0)} \cdot \nabla \rho^{(0)}$

where we used the condition $\nabla \cdot \boldsymbol{B}^{(0)}=0$. Finally, we introduce the variable $s= \pm c_{\mathrm{A}}^{(0)} t$, which represents the distance travelled by the perturbation along the ray. Thus

$\frac{\mathrm{d} \delta U}{\mathrm{~d} s}=\mp \frac{\delta U}{2 \rho^{(0)}} \boldsymbol{b}^{(0)} \cdot \nabla \rho^{(0)}$

The spatial scale where volume $\delta U$ varies is given by $l_{\delta U} \sim 2 l_{\rho}$, where $l_{\rho}$ is the scale of the background density. The latter quantity can be estimated by assuming equilibrium between gravity and pressure gradient and a uniform temperature $T^{(0)}$ :

$l_{\delta U} \sim \frac{2 \kappa_{\mathrm{B}} T^{(0)}}{m_{\mathrm{p}} g}$

where $\kappa_{\mathrm{B}}$ is the Boltzmann constant, $m_{\mathrm{p}}$ the proton mass, $g \simeq$ $2.74 \times 10^{4} \mathrm{~cm} \mathrm{~s}^{-2}$ the surface gravity of the Sun, and $T^{(0)} \simeq$ $1.5 \times 10^{6} \mathrm{~K}$. Using these values, we find $l_{\delta U} \sim 9 \times 10^{4} \mathrm{~km}$. As we shall see, the dynamics of Alfvénic perturbations take place on scales much shorter than $l_{\delta U}$. Then, in Eq. (17) we can safely assume that $\nabla \rho^{(0)} \sim 0$, implying that the volume $|\delta U|$ of perturbation elements remains approximately constant along the rays.

In order to illustrate the dynamics of the perturbation that follows from the above-discussed properties, let us consider an element of perturbation in the form of a infinitesimal cube; the base of the cube lies on the surface of a wave front and has sides $\delta \boldsymbol{x}^{A}$ and $\delta \boldsymbol{x}^{B}$; the other side $\delta \boldsymbol{x}^{C}$ is along the local wavevector $\boldsymbol{k}$. The area of the base is $\delta A=\left|\delta \boldsymbol{x}^{A} \times \delta \boldsymbol{x}^{B}\right|$. When this perturbation element propagates along the rays, it is rotated and stretched, so that the cube is changed into a parallelepiped with non-orthogonal sides. However, during propagation the base keeps lying on the wavefront ( $\delta \boldsymbol{x}^{A}$ and $\delta \boldsymbol{x}^{B}$ both keeping perpendicular to $\boldsymbol{k}$ ), while the volume $|\delta U|$ remains constant. If the perturbation element is stretched in directions parallel to the wavefront so that the area $\delta A$ of the base increases, then it will be compressed in the direction perpendicular to the wavefront, and this will correspond to an increase in the wavevector $k$. Thus, in order to locally build small-scale structures up in the perturbation, it is necessary that points belonging to the same wavefront locally move apart from each other. Since each point in the perturbation propagates along the magnetic field lines, the possibility of an Alfvénic perturbation developing small scales (locally increasing the wavevector $k$ ) is related to the rate of divergence of pairs of field lines.

This property can be quantified by means of the Kolmogorov entropy of magnetic lines (Rechester et al. 1979; Bickerton 1997). Let us suppose that a given infinitesimal vector difference $\delta \boldsymbol{x}$ increases or decreases exponentially with the distance $s$ along a given ray:

$|\delta \boldsymbol{x}(s)|=|\delta \boldsymbol{x}(s=0)| \exp (\lambda s)$

where $\lambda$ is the growth rate and $s=0$ corresponds to an arbitrary initial position along the ray path. In this equation and in what 
follows, we use the variable $s$ instead of time $t$ because we are interested in evaluating the distance travelled by the perturbation before its wavevector increases significantly. The growth rate $\lambda$ depends on the direction of the particular vector difference $\delta \boldsymbol{x}$ considered. We are interested in evaluating the highest growth rate with respect to all the possible directions of $\delta \boldsymbol{x}(s=0)$. This highest growth rate represents the Kolmogorov entropy $H$, which is defined by

$H=\max \lim _{|\delta \boldsymbol{x}(s=0)| \rightarrow 0} \lim _{s \rightarrow+\infty} \frac{1}{s} \ln \left[\frac{|\delta \boldsymbol{x}(s)|}{|\delta \boldsymbol{x}(s=0)|}\right]$.

If the growth of $|\delta \boldsymbol{x}|$ proceeds exponentially with $s$, the limit for $s \rightarrow+\infty$ gives a finite value. In contrast, if the growth of $|\delta \boldsymbol{x}|$ is slower than exponential, then the limit is vanishing. The latter situation is verified in $2 \mathrm{D}$ configurations giving rise to phasemixing, where the stretching process of Alfvénic perturbations is linear with $s$. In that case, $|\delta \boldsymbol{x}|$ grows proportional to $s$ and the Kolmogorov entropy $H$ is equal to zero. The Kolmogorov entropy corresponds to the largest of the three Lyapunov exponents. In a dynamical system where the volumes $\delta V$ of the phase space are conserved along the trajectories, the sum of Lyapunov exponents is equal to zero; then, Lyapunov exponents cannot all be negative. In our case, where the conservation of the volumes $\delta V$ is verified, this implies that $H \geq 0$.

To evaluate the Kolmogorov entropy of magnetic lines $H$ in our configuration, we used a method derived from the algorithm of Wolf et al. (1985) for calculating Lyapunov exponents. This procedure considers a 3D dynamical system where vector differences exponentially increase according to the law (19). Among all possible initial vectors, there is a vector denoted by $\delta \boldsymbol{x}_{\max }(s=0)$, which corresponds to the maximum growth rate $H$. Three arbitrary initial vectors $\delta \boldsymbol{x}^{(m)}(s=0)$, with $m=1,2,3$, orthogonal to each other are chosen. At least one among these vectors, say $\delta \boldsymbol{x}^{(j)}(s=0)$, has a nonvanishing component in the direction of the fastest growth given by $\delta \boldsymbol{x}_{\max }(s=0)$. With increasing $s$ this component increases exponentially, faster than any other component. Correspondingly, the vector $\delta \boldsymbol{x}^{(j)}(s)$ tends to become aligned with $\delta \boldsymbol{x}_{\max }(s)$. Thus, an estimation of the Kolmogorov entropy $H$ can be obtained by calculating the growth rate $\lambda^{(j)}$ of $\delta \boldsymbol{x}^{(j)}(s)$. Since the directions of the three vectors $\delta \boldsymbol{x}^{(m)}(s=0)$ are arbitrarily chosen, the best estimation of $H$ is the maximum among the growth rates $\lambda^{(m)}$ of these three vectors:

$H \simeq \max _{m=1,2,3} \lambda^{(m)}$.

The growth rates $\lambda^{(m)}$ are estimated by calculating the time evolution of the vectors $\delta \boldsymbol{x}^{(m)}(s)$, which is obtained by numerically solving the evolution equations, derived from Eqs. (11) and (15):

$\frac{\mathrm{d} x_{i}}{\mathrm{~d} s}=b_{i}^{(0)}$

$\frac{\mathrm{d} \delta x_{i}^{(m)}}{\mathrm{d} s}=\frac{1}{c_{\mathrm{A}}^{(0)}} \frac{\partial c_{\mathrm{A} i}^{(0)}}{\partial x_{n}} \delta x_{n}^{(m)}, \quad m=1,2,3$.

Starting from an initial position $\boldsymbol{x}_{0}=\boldsymbol{x}(s=0)$ and an initial vector difference $\delta \boldsymbol{x}_{0}^{(m)}=\delta \boldsymbol{x}^{(m)}(s=0)$, Eqs. (22) and (23) are numerically integrated by a 5th-order Runge-Kutta method, up to the distance $s_{1}=N \Delta s$, where $\Delta s$ is the integration step. Due to the linearity of Eq. (23), the evolution of the vector difference $\delta \boldsymbol{x}^{(m)}(s)$ is independent of its initial magnitude. For this reason, we choose the initial vector differences as unit vectors: $\left|\delta \boldsymbol{x}_{0}^{(m)}\right|=1$. An estimation of the growth rates $\lambda^{(m)}$ is then obtained by the following average over the length $s_{1}$ :

$$
\lambda^{(m)} \sim \frac{\sum_{n=1}^{N} \ln \left|\delta \boldsymbol{x}^{(m)}\left(s_{n}\right)\right|}{\sum_{n=1}^{N} s_{n}}
$$

where $s_{n}=n \Delta s$. In order for the solution of Eqs. (22) and (23) to be accurate, the value of the integration step $\Delta s$ must be much lower than the length scale $L_{0}$ of variation for the background quantities. We use $\Delta s / L_{0}=3.5 \times 10^{-2}$. If we look for a local estimation of $H$, the number $N$ of steps cannot be too large: we used $N=10$, so that the length used to estimate $H$ is $s_{1}=0.35 L_{0}$.

\section{Equilibrium structure}

Fast generation of small scales in Alfvénic perturbations can be quantified by means of the Kolmogorov entropy of magnetic lines $H$. To illustrate this method, we have chosen a particular equilibrium structure. However, the same technique could be applied to other equilibria: for instance, a coronal magnetic field obtained by extrapolation from photospheric measurements.

The background Alfvén velocity $\boldsymbol{c}_{\mathrm{A}}^{(0)}(\boldsymbol{x})$ is determined both by $\rho^{(0)}(\boldsymbol{x})$ and by $\boldsymbol{B}^{(0)}(\boldsymbol{x})$. The simplest choice is to assume uniform density, thus we used $\rho^{(0)}(\boldsymbol{x})=1$ (in normalized units). We expect that the more complex the topology of the background magnetic field, the more efficient the generation of small scales in Alfvénic perturbations. Magnetograms of the photospheric magnetic field show that a quiet-Sun region is characterized by a complex pattern formed by many smaller areas of the two magnetic polarities, at different spatial scales and irregularly intermixed (Schrijver et al. 1997). This gives rise to a complex topology of the magnetic field in the corona, above such a region. For this reason, we chose to use an equilibrium magnetic field that represents a model for the coronal magnetic field above a quietSun region. This model field has been introduced by Nakagawa \& Raadu (1972) and used in Paper I to evaluate the energy dissipation of Alfvén waves in coronal magnetic fields. The assumptions are:

1) within the cold plasma approximation $(\beta \sim 0)$, an equilibrium magnetic field $\boldsymbol{B}^{(0)}$ satisfies the force-free condition

$\nabla \times \boldsymbol{B}^{(0)}=\alpha \boldsymbol{B}^{(0)}$,

which is derived from the momentum equation (3) in the static case $(\boldsymbol{v}=0)$. In this equation we assume that $\alpha$ is constant. The constant- $\alpha$ condition is quite restrictive, but, on the other hand, it greatly simplifies the calculation of $\boldsymbol{B}^{(0)}$;

2) we consider a planar geometry and a cartesian reference frame, with the base of the corona in the $x y$-plane and the $z$-axis directed vertically upward. This implies that a relatively small portion of the Corona is described, so that spherical curvature effects can be neglected;

$3)$ we assume statistical homogeneity in the horizontal directions $(x$ and $y)$. No dominant large-scale structures are present; on the contrary, the equilibrium magnetic field distribution is characterized by several irregular regions of the two magnetic polarities, randomly distributed. To model this kind of configuration, periodicity in the $x$ and $y$ directions is assumed, over a periodicity length $L$, which is used as the 
unit length. Thus the spatial domain $D$ is an infinitely tall parallelepiped with a unitary square basis: $D=\{x, y, z\}=$ $[0,1] \times[0,1] \times[0,+\infty)$. Moreover, the magnetic field is characterized by a typical horizontal scale $L_{0}$. Statistical homogeneity is reproduced when $L_{0} \ll L$ (Pommois et al. 1998). We used $L_{0}=L / 4$. Comparing with magnetograms in quietSun regions (e.g., Schrijver et al. 1997), we estimate $L_{0} \sim$ $2.5-3 \times 10^{4} \mathrm{~km}$, giving a unit length $L \sim 1-1.2 \times 10^{5} \mathrm{~km}$. In normalized units, the typical horizontal scale is $l_{0}=L_{0} / L=$ 0.25

4) the magnetic field vanishes in the limit $z \rightarrow \infty$ (Nakagawa $\&$ Raadu 1972). This implies that the magnetic energy inside the domain $D$ is finite (Alissandrakis 1981).

Under the above assumptions, the equilibrium magnetic field $\boldsymbol{B}^{(0)}$ can be written as a superposition of Fourier harmonics, in the following form (Paper I):

$$
\begin{aligned}
B_{x}^{(0)}(x, y, z)= & 2 \sum_{\kappa_{0} \leq \kappa \leq \kappa_{\max }, \kappa_{x} \geq 0}\left(\frac{\kappa_{x} \sqrt{\kappa^{2}-\alpha^{2}}}{\kappa^{2}}-\frac{\kappa_{y} \alpha}{\kappa^{2}}\right)\left|a\left(\kappa_{x}, \kappa_{y}\right)\right| \\
& \times \cos \left[\kappa_{x} x+\kappa_{y} y+\phi\left(\kappa_{x}, \kappa_{y}\right)\right] \exp \left(-\sqrt{\kappa^{2}-\alpha^{2} z}\right) \\
B_{y}^{(0)}(x, y, z)= & 2 \sum_{\kappa_{0} \leq \kappa^{\prime} \kappa_{\max }, \kappa_{x} \geq 0}\left(\frac{\kappa_{y} \sqrt{\kappa^{2}-\alpha^{2}}}{\kappa^{2}}+\frac{\kappa_{x} \alpha}{\kappa^{2}}\right)\left|a\left(\kappa_{x}, \kappa_{y}\right)\right| \\
\times & \cos \left[\kappa_{x} x+\kappa_{y} y+\phi\left(\kappa_{x}, \kappa_{y}\right)\right] \exp \left(-\sqrt{\kappa^{2}-\alpha^{2}} z\right) \\
B_{z}^{(0)}(x, y, z)= & -2 \sum_{\kappa_{0} \leq \kappa \leq \kappa_{\max }, \kappa_{x} \geq 0}\left|a\left(\kappa_{x}, \kappa_{y}\right)\right| \\
& \times \sin \left[\kappa_{x} x+\kappa_{y} y+\phi\left(\kappa_{x}, \kappa_{y}\right)\right] \exp \left(-\sqrt{\kappa^{2}-\alpha^{2}} z\right)
\end{aligned}
$$

where $\kappa_{x}=2 \pi n_{x}, \kappa_{y}=2 \pi n_{y}\left(n_{x}\right.$ and $n_{y}$ integers $)$, and $\kappa=$ $\left(\kappa_{x}^{2}+\kappa_{y}^{2}\right)^{1 / 2}$ is the horizontal wavenumber of the different harmonics. Each harmonic is periodic in the $x$ and $y$ directions, and it exponentially decreases with increasing $z$, with the rate $\gamma_{z}=\sqrt{\kappa^{2}-\alpha^{2}}$.

According to the induction Eq. (4), the inhomogeneous $\boldsymbol{B}_{0}(\boldsymbol{x})$ should evolve in time due to resistive diffusion. However, since the magnetic diffusivity $\lambda$ is extremely small in the corona, the resistive diffusion time $t_{\lambda}=\left(L_{0}^{2} / \lambda\right)\left(\kappa_{0} / \kappa_{\max }\right)^{2}$ of the magnetic structure is much longer than the evolution time of perturbations. Then, we can neglect resistive diffusion and consider $\boldsymbol{B}_{0}(\boldsymbol{x})$ as static.

Since the equilibrium structure (26) represents a generic equilibrium structure above a quiet-Sun region, the parameters in Eqs. (26) have been chosen in the following way.

1) The amplitudes $\left|a\left(\kappa_{x}, \kappa_{y}\right)\right|$ depend only on the modulus $\kappa$ of the horizontal wavevector, according to a power law:

$\left|a\left(\kappa_{x}, \kappa_{y}\right)\right|=c \kappa^{-\mu} \quad$ for $\kappa_{0} \leq \kappa \leq \kappa_{\max }$

where $\mu$ is the spectral index and $c$ a normalization constant. We use $\mu=4 / 3$, corresponding to a Kolmogorov spectrum for the magnetic fluctuations at the coronal base (Paper I). The constant $c$ is determined by requiring that the rms magnetic field at $z=0$ is $\left[\left\langle B^{(0)^{2}}\right\rangle_{x y}(z=0)\right]^{1 / 2}=1$.

2) The minumum wavenumber is $\kappa_{0}=2 \pi / l_{0}=8 \pi$, corresponding to the dominant horizontal length $l_{0}$. The maximum wavenumber has been chosen as $\kappa_{\max }=20 \pi$; with this choice, each component of the equilibrium magnetic field (26) corresponds to the superposition of 133 harmonics, which gives a complex magnetic structure, but computation times are not exceedingly long.

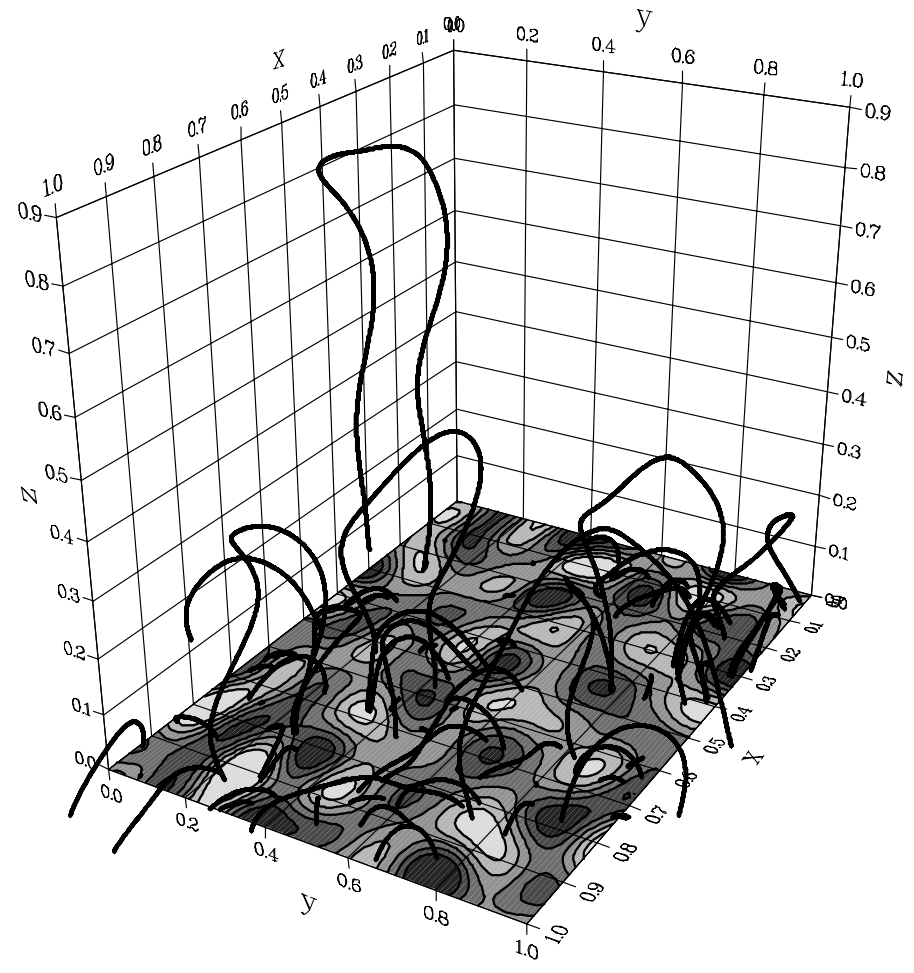

Fig. 1. A contour plot of the vertical component $B_{z}^{(0)}$ of the equilibrium magnetic field, shown in the $x y$ plane. Dark or light tones correspond to positive or negative values of $B_{z}^{(0)}$, respectively. The trajectories of 70 magnetic lines are also shown. The starting point of each magnetic line is at the base $z=0$, randomly chosen inside the periodicity domain $D$.

3) Parameter $\alpha$ determines the current density $\boldsymbol{j}^{(0)}=(c / 4 \pi) \nabla \times$ $\boldsymbol{B}^{(0)}$ to the equilibrium magnetic field $\boldsymbol{B}^{(0)}$ ratio. Moreover, the value of $\alpha$ in our model is bounded by the minimum horizontal wavevector: $\alpha<\kappa_{0}$. We considered two different values for the parameter $\alpha$ : a) $\alpha=0$, corresponding to a potential magnetic field $\left(\boldsymbol{j}^{(0)}=0\right)$; b) $\alpha=7 \pi$, which is close to the upper bound $\kappa_{0}$ and gives a nonvanishing current $\boldsymbol{j}^{(0)}$.

4) Since $\boldsymbol{B}^{(0)}$ does not represent any particular magnetic field, the phases $\left\{\phi\left(\kappa_{x}, \kappa_{y}\right)\right\}$ of harmonics have been randomly chosen in the interval $[0,2 \pi[$.

In Fig. 1 the structure of the equilibrium magnetic field (26) is illustrated for $\alpha=7 \pi$, inside the periodicity domain $D$. The contour plot in the $x y$ plane gives the distribution of the vertical $B_{z}^{(0)}$ component at the base $z=0$; dark or light tones correspond to positive or negative values of $B_{z}^{(0)}$, respectively. It can be seen that several irregular regions of the two magnetic polarities are present, randomly distributed; the resulting pattern is reminiscent of the magnetograms of quiet-Sun regions (Schrijver et al. 1997). The trajectories of 70 magnetic lines are also displayed, with starting points located at the base $z=0$. Each line links two regions of opposite polarity. Most lines are in the form of small arcs standing above a neutral line and are limited to lower altitudes. Lines reaching higher altitudes are more irregular. The topology of the equilibrium magnetic field (26) is quite complex (Paper I), thus it can be expected that an effective generation of small scales takes place in Alfvénic perturbations propagating in a structure like this. 


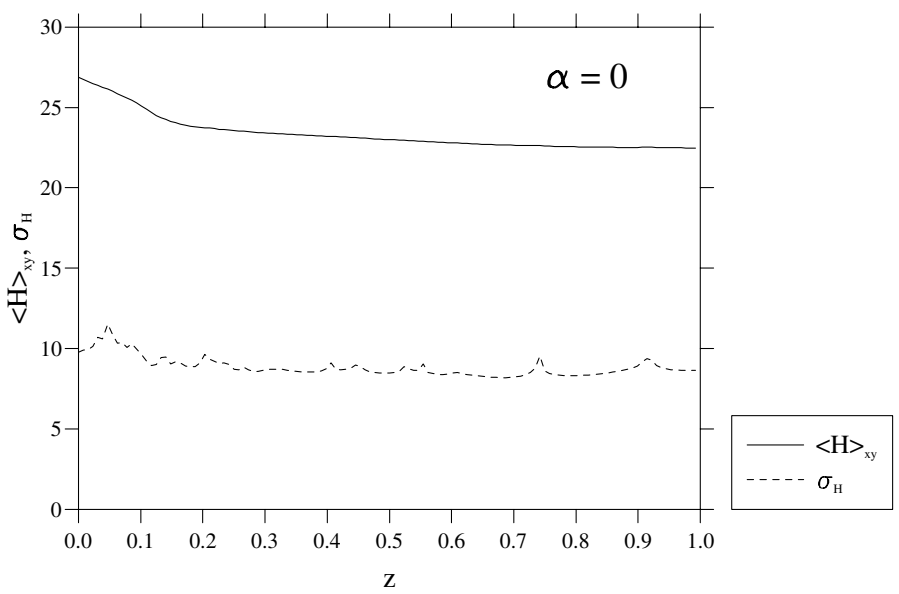

Fig. 2. The average Kolmogorv entropy $\langle H\rangle_{x y}$ (full line) and the rms deviation $\sigma_{H}$ (dashed line) as functions of the altitude $z$, calculated for $\alpha=0$ (potential field).

\section{Calculation of the Kolmogorov entropy of magnetic lines}

We applied the method described in Sect. 3 to calculate the distribution of $H$ in the considered equilibrium structure. The values of $H$ have been calculated in a large number of locations forming a regular 3D grid inside the periodicity domain $D: \boldsymbol{x}_{0}=((i-1) \Delta x,(j-1) \Delta x,(k-1) \Delta x)$, with $i, j, k=1, \ldots, M+1$ and $\Delta x=1 / M$ the grid step. We used $M=128$ corresponding to more than $2 \times 10^{6}$ grid points. Such a grid covers the whole extension of the periodicity domain in the horizontal directions, and it reaches the altitude $z=1$. Starting from each grid point, we solved Eqs. (22), (23) along the short distance $s_{1}$ following the evolution of two different terns of initial vector differences $\delta \boldsymbol{x}: \delta \boldsymbol{x}^{(1)}=(1,0,0) ; \delta \boldsymbol{x}^{(2)}=(0,1,0) ; \delta \boldsymbol{x}^{(3)}=(0,0,1) ; \delta \boldsymbol{x}^{(4)}=$ $(1 / \sqrt{3}, 1 / \sqrt{3}, 1 / \sqrt{3}) ; \delta \boldsymbol{x}^{(5)}=(-2 / \sqrt{6}, 1 / \sqrt{6}, 1 / \sqrt{6}) ; \delta \boldsymbol{x}^{(6)}=$ $(0,1 / \sqrt{2},-1 / \sqrt{2})$. The local value of the Kolmogorov entropy is evaluated as the maximum growth rate $H=\max \gamma^{(m)}, m=$ $1, \ldots, 6$, where each $\gamma^{(m)}$ is calculated by Eq. (24). Using 6 vector differences $\delta \boldsymbol{x}$ instead of 3 increases the probability that one of these vectors is initially close to the direction of maximum growth $\delta \boldsymbol{x}_{\max }(s=0)$, giving a better evaluation of $H$. However, at the end of the integration of Eqs. (22), (23), none of the six unit vectors $\delta \boldsymbol{x}^{(m)}$ will be exactly aligned along the maximum growth direction. Then, the value $H$ of the Kolmogorov entropy is underestimated.

Since the equilibrium structure is statistically homogeneous in the horizontal directions, we calculate the value of $H$ averaged in the $x$ and $y$ directions

$\langle H\rangle_{x y}\left(z_{k}\right)=\frac{1}{M^{2}} \sum_{i, j=1}^{M} H\left(x_{i}, y_{j}, z_{k}\right)$

and the corresponding rms deviation

$\sigma_{H}\left(z_{k}\right)=\left[\frac{1}{M^{2}} \sum_{i, j=1}^{M}\left[H\left(x_{i}, y_{j}, z_{k}\right)-\langle H\rangle_{x y}\left(z_{k}\right)\right]^{2}\right]^{1 / 2}$.

The values of $\langle H\rangle_{x y}$ and of $\sigma_{H}$ are plotted as functions of $z_{k}$ for $\alpha=0$ (Fig. 2) and for $\alpha=7 \pi$ (Fig. 3). In both cases the value of the average Kolmogorov entropy is the highest at the coronal base $z=0$; it decreases slightly up to the altitude $z \simeq 0.15$. At higher altitudes $\langle H\rangle_{x y}$ remains almost constant. We recall that in

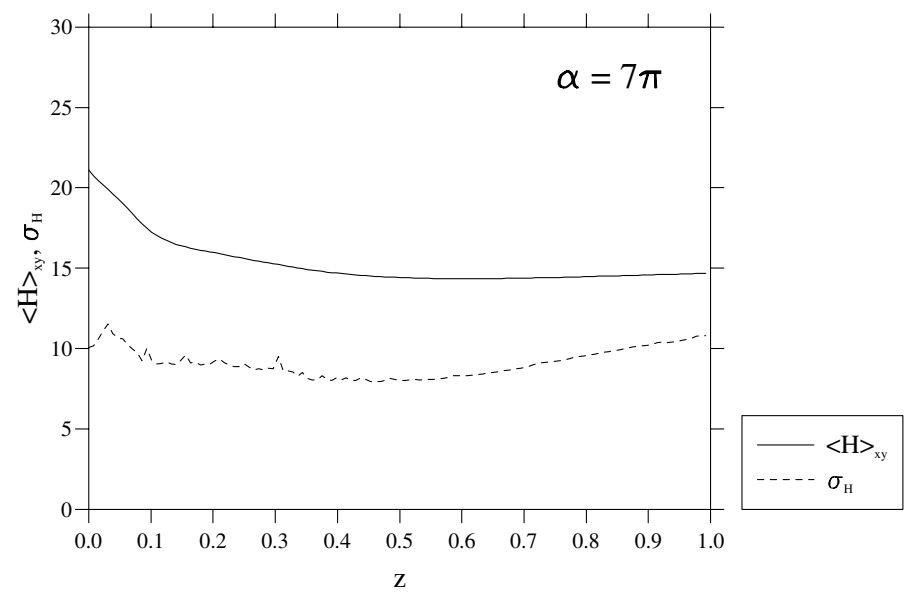

Fig. 3. The same as in Fig. 2, calculated for $\alpha=7 \pi$ (field with current).

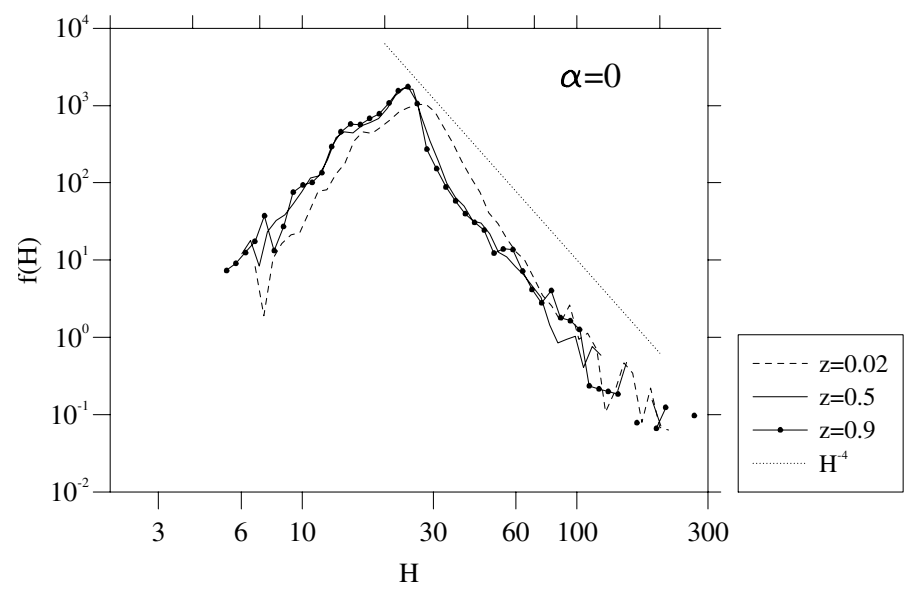

Fig. 4. The distribution $f(H)$ of the Kolmogorov entropy of magnetic lines, calculated at $z=0.02$ (dashed line), $z=0.5$ (full line), $z=0.9$ (full line with dots), calculated for $\alpha=0$. The dotted line indicates a slope proportional to $H^{-4}$.

these units the typical size of the magnetic structures in the horizontal direction is $\sim 0.25$. In the case of potential field ( $\alpha=0$ ), the average Kolmogorov entropy is in the range $\langle H\rangle_{x y} \sim 23-27$, while $\langle H\rangle_{x y} \sim 15-21$ in the case of a magnetic field with strong current $(\alpha=7 \pi)$. The rms deviation $\sigma_{H}$ decreases slightly with the altitude $z$ for the potential field, being $\sigma_{H} \simeq 9-10$. For $\alpha=7 \pi$, the values of $\sigma_{H}$ are in the same range, but they decrease with $z$ at low altitudes and increase again at higher altitudes.

When comparing Figs. 2 and 3, it can be seen that on average the ratio $\sigma_{H} /\langle H\rangle_{x y}$ is higher for the magnetic field with current. Then, the distribution of the values of Kolmogorov entropy is wider in the case of a magnetic field with a strong current. We calculated the distribution $f(H)$ in both cases for three different altitudes: close to the coronal base $(z=0.02)$, at an intermediate altitude $(z=0.5)$, and at high altitudes $(z=0.9)$. The distributions $f(H)$ are plotted in Fig. 4 for $\alpha=0$ and in Fig. 5 for $\alpha=7 \pi$. In all cases a long power-law tail is present with a slope close to -4 for $\alpha=0$, being less steep for $\alpha=7 \pi$. The tail in the distribution $f(H)$ is described better by a power law for higher values of $\alpha$, and at intermediate and high altitudes. Closer to the coronal base, the tail in $f(H)$ is steeper. The maximum value of $H$ in these distributions is 15-20 times higher than the mean value: e.g., for $\alpha=7 \pi$ the highest value of $H$ is $\sim 300$. However, this maximum value is probably determined by the finite number of grid points; we expect that, if using a smaller grid step $\Delta x$, the 


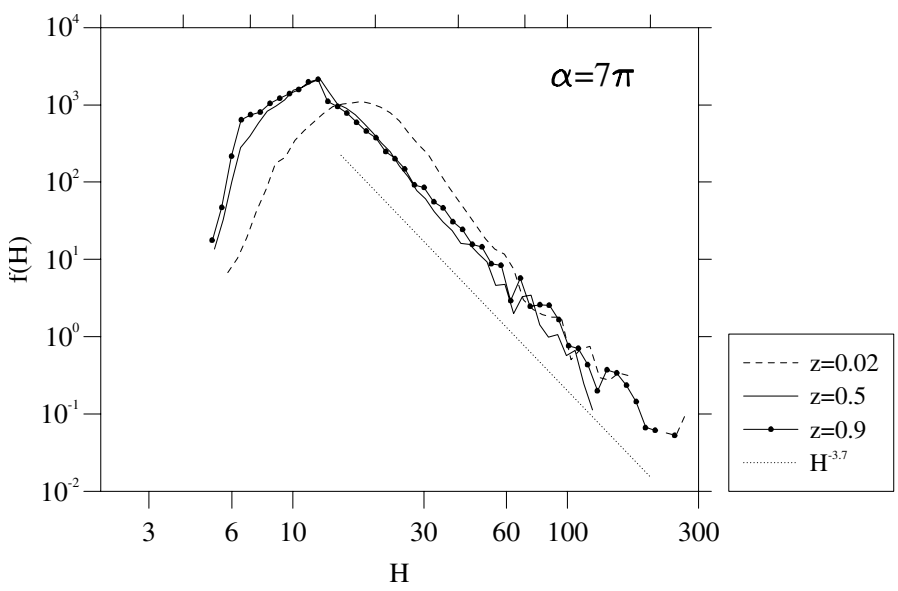

Fig. 5. The distribution $f(H)$ of the Kolmogorov entropy of magnetic lines, calculated at $z=0.02$ (dashed line), $z=0.5$ (full line), $z=0.9$ (full line with dots), calculated for $\alpha=7 \pi$. The dotted line indicates a slope proportional to $H^{-3.7}$.

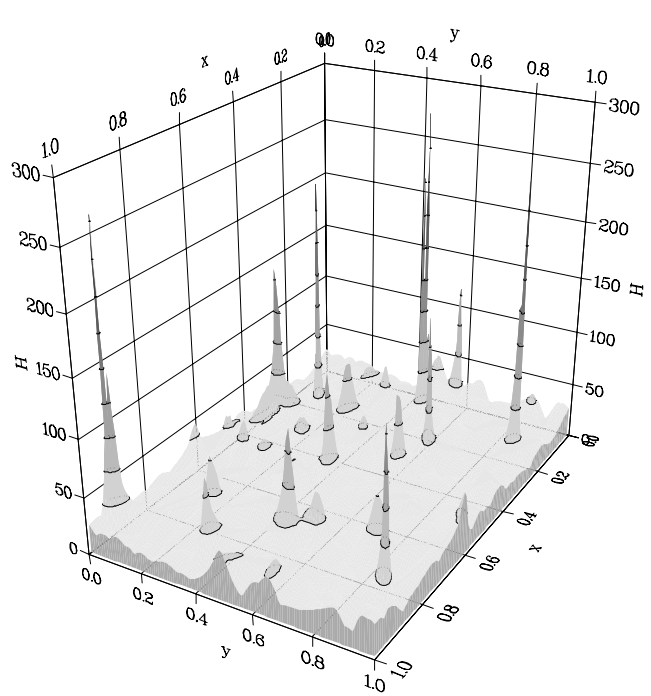

$$
\alpha=7 \pi
$$$$
\mathrm{z}=0.02
$$

Fig. 6. The Kolmogorov entropy of magnetic lines $H$ calculated at $z=$ 0.02 for $\alpha=7 \pi$.

distribution $f(H)$ would extend to higher values of $H$. This suggests that there could be locations inside the magnetic structure where the Kolmogorov entropy $H$ diverges to $+\infty$. Around such locations the rate of small-scale generation is much higher than in the rest of the magnetic structure.

To illustrate this behavior, in Fig. 6 we plotted the values of the Kolmogorov entropy $H$ as a function of $x$ and $y$, at a fixed altitude $z=0.02$ and for $\alpha=7 \pi$. The plot is characterized by several sharp peaks that rise much higher than the average value. The high values of $H$ corresponding to the tail of $f(H)$ are localized in well-defined locations inside the equilibrium magnetic field. A behavior similar to that shown in Fig. 6 has also been found at intermediate and high altitudes $z$, as well as for $\alpha=0$.

Figure 7 illustrates the 3D spatial distribution of locations where $H$ is particularly large. This figure represents the whole spatial domain and each dot corresponds to a point where the value of $H$ is higher than $\langle H\rangle_{x y}+3 \sigma_{H}$ at each given altitude $z$ (case $\alpha=7 \pi$ ). It can be seen that locations where $H$ is particularly high are spatially organized in straight filaments that are mainly oriented in the vertical direction. This is verified especially at intermediate and high altitudes; at low altitudes the

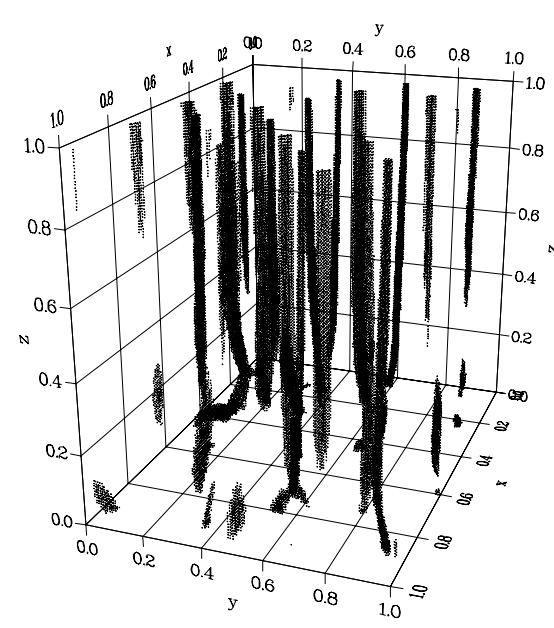

$\alpha=7 \pi$

$\mathrm{H}><\mathrm{H}>_{\mathrm{xy}}+3 \sigma_{\mathrm{H}}$

Fig. 7. The 3D distribution of high Kolmogorov entropy locations inside the spatial domain, for $\alpha=7 \pi$. Each dot corresponds to a location where $H>\langle H\rangle_{x y}+3 \sigma_{H}$.

filaments can also be curved or they can have more complex shapes. A similar behavior has also been found for the potential magnetic field $(\alpha=0)$.

The spatial distribution of high- $H$ locations is related to the local topological properties of the equilibrium magnetic field $\boldsymbol{B}^{(0)}$ (Eqs. (26)) in which the decay rate of harmonics is higher for larger horizontal wavenumber $\boldsymbol{\kappa}$. At low altitudes $z$, all the harmonics contribute to $\boldsymbol{B}^{(0)}$, while only the contribution of small wavenumber harmonics survives at high altitudes. Then, the magnetic topology is more complex at low altitudes, while above a given altitude the magnetic structure becomes more ordered. This explains why higher values of the Kolmogorov entropy are found at small $z$, as well as ordered filaments of large $H$ locations at high altitudes.

\section{Magnetic topology at locations of high magnetic-line Kolmogorov entropy}

Since high values of the Kolmogorov entropy of magnetic lines are localized in specific locations, it is useful to characterize the topology of the magnetic field around such locations. Since $\boldsymbol{B}^{(0)}$ is parallel to $\boldsymbol{c}_{\mathrm{A}}^{(0)}$, magnetic lines are equivalent to fieldlines of $\boldsymbol{c}_{\mathrm{A}}^{(0)}$. We expand the vector field $\boldsymbol{c}_{\mathrm{A}}^{(0)}(\boldsymbol{x})$ around a location $\boldsymbol{x}_{0}$. At the first order, we get the expression

$$
\begin{aligned}
c_{\mathrm{A} i}^{(0)}(\boldsymbol{x}) & \simeq c_{\mathrm{A} i}^{(0)}\left(\boldsymbol{x}_{0}\right)+\frac{\partial c_{\mathrm{A} i}^{(0)}}{\partial x_{j}}\left(x_{j}-x_{0 j}\right) \\
& =c_{\mathrm{A} i}^{(0)}\left(\boldsymbol{x}_{0}\right)+\left(S_{i j}+A_{i j}\right)\left(x_{j}-x_{0 j}\right),
\end{aligned}
$$

where

$S_{i j}=\frac{1}{2}\left(\frac{\partial c_{\mathrm{A} i}^{(0)}}{\partial x_{j}}+\frac{\partial c_{\mathrm{A} j}^{(0)}}{\partial x_{i}}\right), \quad A_{i j}=\frac{1}{2}\left(\frac{\partial c_{\mathrm{A} i}^{(0)}}{\partial x_{j}}-\frac{\partial c_{\mathrm{A} j}^{(0)}}{\partial x_{i}}\right)$

are the symmetric and anti-symmetric parts of the matrix $\partial c_{\mathrm{A} i}^{(0)} / \partial x_{j}$, respectively. The matrix $S_{i j}$ has real eigenvalues and mutually orthogonal eigenvectors; then, it can be diagonalized by rotating the reference frame, so that the new axes are parallel to the three eigenvectors of $S_{i j}$. In the new reference frame, matrix $A_{i j}$ remains anti-symmetric and Eq. (30) has the form

$c_{\mathrm{A} i}^{(0)}(\boldsymbol{x}) \simeq c_{\mathrm{A} i}^{(0)}\left(\boldsymbol{x}_{0}\right)+\zeta^{(i)}\left(x_{i}-x_{0 i}\right)+\left[\boldsymbol{R} \times\left(\boldsymbol{x}-\boldsymbol{x}_{0}\right)\right]_{i}$ 
where $\zeta^{(i)}$ is the $i$ th eigenvalue of $S_{i j}$ and $\boldsymbol{R}=\nabla \times \boldsymbol{c}_{\mathrm{A}}^{(0)}$. The components of the vector $\boldsymbol{R}$ form the off-diagonal elements of matrix $A_{i j}$ in the rotated reference frame. When $\rho^{(0)}$ is uniform, $\boldsymbol{R}$ is proportional both to the current density $\boldsymbol{j}^{(0)}=(c / 4 \pi) \nabla \times \boldsymbol{B}^{(0)}$ and to $\boldsymbol{c}_{\mathrm{A}}^{(0)}$ (Eq. (25)):

$\boldsymbol{R}=\frac{1}{c}\left(\frac{4 \pi}{\rho^{(0)}}\right)^{1 / 2} \boldsymbol{j}^{(0)}=\alpha \boldsymbol{c}_{\mathrm{A}}^{(0)}$.

Moreover,

$\zeta^{(1)}+\zeta^{(2)}+\zeta^{(3)}=\nabla \cdot c_{\mathrm{A}}^{(0)}=\frac{\nabla \cdot \boldsymbol{B}^{(0)}}{\left(4 \pi \rho^{(0)}\right)^{1 / 2}}=0$.

Thus, for uniform $\rho^{(0)}$ Eq. (32) reduces to

$c_{\mathrm{A} i}^{(0)}(\boldsymbol{x}) \simeq c_{\mathrm{A} i}^{(0)}\left(\boldsymbol{x}_{0}\right)+\zeta^{(i)}\left(x_{i}-x_{0 i}\right)+\alpha\left[\boldsymbol{c}_{\mathrm{A}}^{(0)}\left(\boldsymbol{x}_{0}\right) \times\left(\boldsymbol{x}-\boldsymbol{x}_{0}\right)\right]_{i}$.

In this case the local structure of the vector field $\boldsymbol{c}_{\mathrm{A}}^{(0)}$ can be described in terms of six quantities: namely, the three components of $\boldsymbol{c}_{\mathrm{A}}^{(0)}\left(\boldsymbol{x}_{0}\right)$ and the three eigenvalues $\zeta^{(i)}$. These eigenvalues are not completely independent, in consequence of condition (34). Equation (35) shows that $\boldsymbol{c}_{\mathrm{A}}^{(0)}$ can be locally considered as the superposition of three contributions: (i) a uniform field representing the value of $c_{\mathrm{A}}^{(0)}$ at the location $\boldsymbol{x}_{0}$; (ii) a term representing stretching along one or two principal axes (those corresponding to positive eigenvalues $\zeta^{(i)}$ ) and a contraction along the remaining axes; and (iii) a term describing a torsion of the lines of $\boldsymbol{c}_{\mathrm{A}}^{(0)}$ around the direction of $\boldsymbol{c}_{\mathrm{A}}^{(0)}\left(\boldsymbol{x}_{0}\right)$ with a torsion rate $R=\alpha\left|\boldsymbol{c}_{\mathrm{A}}^{(0)}\left(\boldsymbol{x}_{0}\right)\right|$. For a potential magnetic field $(\alpha=0)$, the torsion term is vanishing.

We used $c_{\mathrm{A}}^{(0)}\left(\boldsymbol{x}_{0}\right)$, the eigenvalues $\zeta^{(i)}$, and the torsion rate $R$ to characterize the structure of $\boldsymbol{c}_{\mathrm{A}}^{(0)}$ around locations where the Kolmogorov entropy is particularly high. We selected a few points $\boldsymbol{x}_{0}$ at different altitudes $z$ where the value of $H$ is close to the maximum, and we calculated the above defined quantities at those locations. The typical behavior in such points can be summarized as follows:

a) The local value of the Alfvén velocity is much lower than the average value of $c_{\mathrm{A}}^{(0)}$ calculated at the same altitude:

$\left|\boldsymbol{c}_{\mathrm{A}}^{(0)}\left(\boldsymbol{x}_{0}\right)\right| \ll\left\langle\left|\boldsymbol{c}_{\mathrm{A}}^{(0)}\right|\right\rangle_{x y} \equiv \frac{1}{L^{2}} \int\left|\boldsymbol{c}_{\mathrm{A}}^{(0)}\left(x, y, z_{0}\right)\right| \mathrm{d} x \mathrm{~d} y$.

Then, locations with the highest values of the Kolmogorov entropy $H$ are quasi-neutral points for the field $c_{\mathrm{A}}^{(0)}$ and for the magnetic field $\boldsymbol{B}^{(0)}$. In order to show this property, we collected all the gridpoints where $H$ exceeds the value $\langle H\rangle_{x y}+3 \sigma_{H}$, and for each point we calculated the ratio $r_{1}=\left|\boldsymbol{c}_{\mathrm{A}}^{(0)}\left(\boldsymbol{x}_{0}\right)\right| /\left\langle\left|\boldsymbol{c}_{\mathrm{A}}^{(0)}\right|\right\rangle_{x y}$. Then, we calculated the 2D histogram representing the distribution of such points with respect to the values of $H$ and $r_{1}$, which is represented in Fig. 8 in logarithmic scale in the case $\alpha=7 \pi$. It can be seen that the ratio $\left|\boldsymbol{c}_{\mathrm{A}}^{(0)}\left(\boldsymbol{x}_{0}\right)\right| /\left\langle\left|\boldsymbol{c}_{\mathrm{A}}^{(0)}\right|\right\rangle_{x y}$ systematically decreases for increasing $H$. A similar behavior has also been found in the case $\alpha=0$.

b) Comparing the highest eigenvalue $\zeta_{\max }=\max \left\{\zeta^{(1)}, \zeta^{(2)}, \zeta^{(3)}\right\}$ and the torsion rate $R$ at location where $H$ is very large, we find that $\zeta_{\max } \gg R$. Thus, at points where the Komogorov entropy is high, the dilatation term in Eq. (32) largely prevails over the torsion term. To show this property, we calculated the ratio $r_{2}=\zeta_{\max } / R$ for all gridpoints where

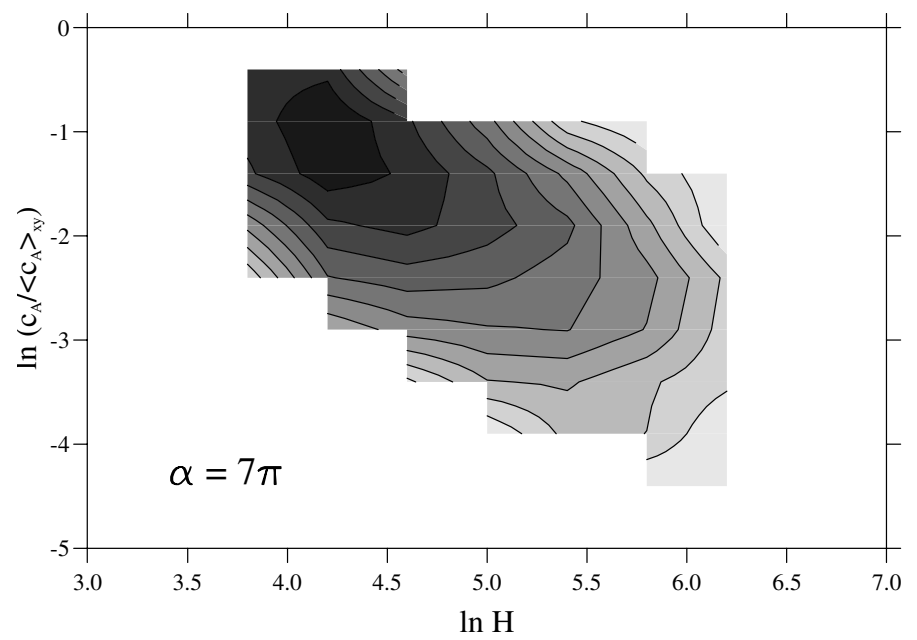

Fig. 8. A $2 \mathrm{D}$ histogram obtained by selecting the gridpoints where $H>\langle H\rangle_{x y}+3 \sigma_{H}$. The horizontal and the vertical axes represent the value of $\ln H$ and of $\ln \left(\left|\boldsymbol{c}_{\mathrm{A} 0}\left(\boldsymbol{x}_{0}\right)\right| /\left\langle\left|\boldsymbol{c}_{\mathrm{A} 0}\right|\right\rangle_{x y}\right)$, respectively, for each selected gridpoint. The histogram is plotted on a logarithmic scale, in the case $\alpha=7 \pi$.

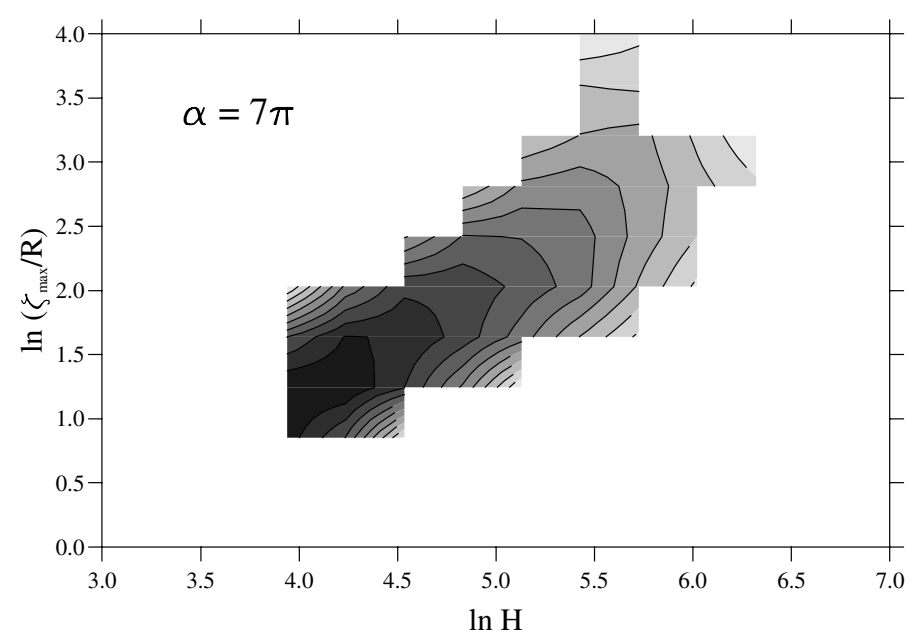

Fig. 9. A 2D histogram obtained by selecting the gridpoints where $H>$ $\langle H\rangle_{x y}+3 \sigma_{H}$. The horizontal and the vertical axes represent the value of $\ln H$ and of $\ln \left(\zeta_{\max } / R\right)$, respectively, for each selected gridpoint. The histogram is plotted on a logarithmic scale, in the case $\alpha=7 \pi$.

$H>\langle H\rangle_{x y}+3 \sigma_{H}$. A 2 D histogram representing the distribution of these points with respect to the values of $H$ and $r_{2}$ is displayed in Fig. 9.

It appears that higher values of $H$ correspond to higher values of the ratio $\zeta_{\max } / R$. Then, locations where small scales are generated more quickly are nearly current-free. This property has been verified for the case $\alpha=7 \pi$. Of course, the torsion rate $R$ is vanishing for $\alpha=0$, so these considerations do not apply.

c) Comparing the highest eigenvalue $\zeta_{\max }$ and the lowest (negative) one $\zeta_{\min }$ at high $H$ locations, we found that $\left|\zeta_{\max }\right| \sim$ $\left|\zeta_{\min }\right|$, while the intermediate eigenvalue $\left|\zeta_{\text {int }}\right| \ll\left|\zeta_{\max }\right|,\left|\zeta_{\min }\right|$. Thus, the spatial structure of $\boldsymbol{c}_{\mathrm{A}}^{(0)}$ is quasi-2D and the coordinate that can be ignored is in the direction of the eigenvector $\boldsymbol{v}_{\text {int }}$ corresponding to the eigenvalue $\zeta_{\text {int }}$.

The above properties indicate that at locations where the Kolmogorov entropy $H$ is very high, the topology of both the vector field $\boldsymbol{c}_{\mathrm{A}}^{(0)}$ and of the magnetic field $\boldsymbol{B}^{(0)}$ corresponds to a quasi-neutral quasi-2D X-point. This can be seen in Figs. 10 


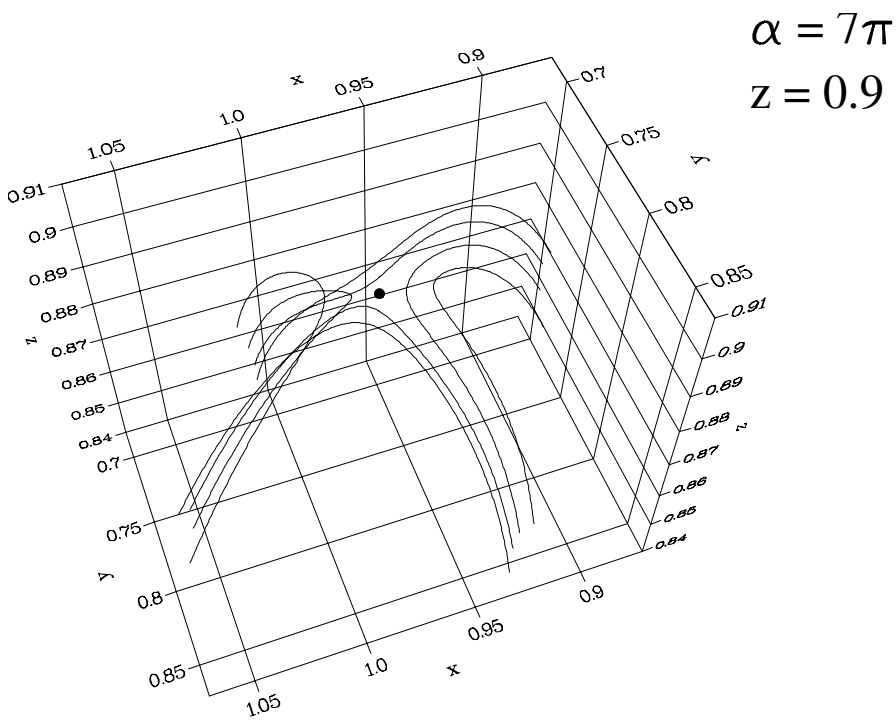

Fig. 10. Fieldlines of $\boldsymbol{c}_{\mathrm{A}}^{(0)}$ around the point $\boldsymbol{x}_{0}=(0.97,0.77,0.90)$, for $\alpha=7 \pi$. The point $x_{0}$ is represented by a dot. The value of the Kolmogorov entropy at that location is $H\left(x_{0}\right)=265.7$.

and 11 where we represent the fieldlines of $\boldsymbol{c}_{\mathrm{A}}^{(0)}$ around two locations with high $H$, for $\alpha=7 \pi$, at altitudes $z=0.9$ and $z=0.05$. A similar pattern was also found for the potential field $(\alpha=0)$. The fieldlines represented in Figs. 10 and 11 were drawn in the following way. We chose a location $\boldsymbol{x}_{1}$ (close to $\boldsymbol{x}_{0}$ ) such that the difference $\boldsymbol{x}_{1}-\boldsymbol{x}_{0}$ is parallel (or antiparallel) to the eigenvector $\boldsymbol{v}_{\min }$ or $\boldsymbol{v}_{\max }$ corresponding to the eigenvalues $\zeta_{\min }$ or to $\zeta_{\max }$, respectively. Starting from $\boldsymbol{x}_{1}$, we followed the corresponding fieldline moving in the directions of $\pm \boldsymbol{c}_{\mathrm{A}}^{(0)}$. As a result, in the vicinity of $x_{0}$, the fieldlines of Figs. 10 and 11 roughly lie in the plane that contains $\boldsymbol{x}_{0}$ and that is parallel to $\boldsymbol{v}_{\min }$ and $\boldsymbol{v}_{\max }$. The largest variations of $\boldsymbol{c}_{\mathrm{A}}^{(0)}$ are in that plane, while, moving in the perpendicular direction (corresponding to $\boldsymbol{v}_{\text {int }}$ ), the field $\boldsymbol{c}_{\mathrm{A}}^{(0)}$ remains almost constant. Then, the $\mathrm{X}$ pattern of fieldlines in Figs. 10 and 11 can be translated along the direction of $\boldsymbol{v}_{\text {int }}$, remaining nearly unchanged. This minimum variance direction is almost vertical in Fig. 10, while it is almost horizontal in Fig. 11.

From these considerations, we can deduce the 3D structure of the field $c_{\mathrm{A}}^{(0)}$ around locations where the Kolmogorov entropy $H$ is very high: there are two surfaces (separatrices) that intesect along a line (separator) (e.g., Priest \& Titov 1996; Priest 2001). Fieldlines on the two sides of one separatrix move away from each other, but eventually converge toward the other separatrix. The X-point (dot) in Figs. 10 and 11 is one point on a separator line. Locations where $H$ is locally maximum correspond to the separator line. This also explains the filamentary structure of Fig. 7, where filaments correspond to separators in the magnetic structure.

\section{Dissipation and fieldline topology}

The points where the rate of small-scale generation in Alfvénic perturbations is particularly high are distributed around the separator lines of the equilibrium magnetic structure which in turn, are at the intersection of the separatrix surfaces. Let us consider an element of an Alfvénic perturbation that propagates along a fieldline approaching a separator, like the lines represented in Figs. 10 or 11: when the perturbation approaches the separator, the growth rate of the wavevector $k$ increases strongly. If $k$ has become large enough, the perturbation is dissipated. Otherwise,

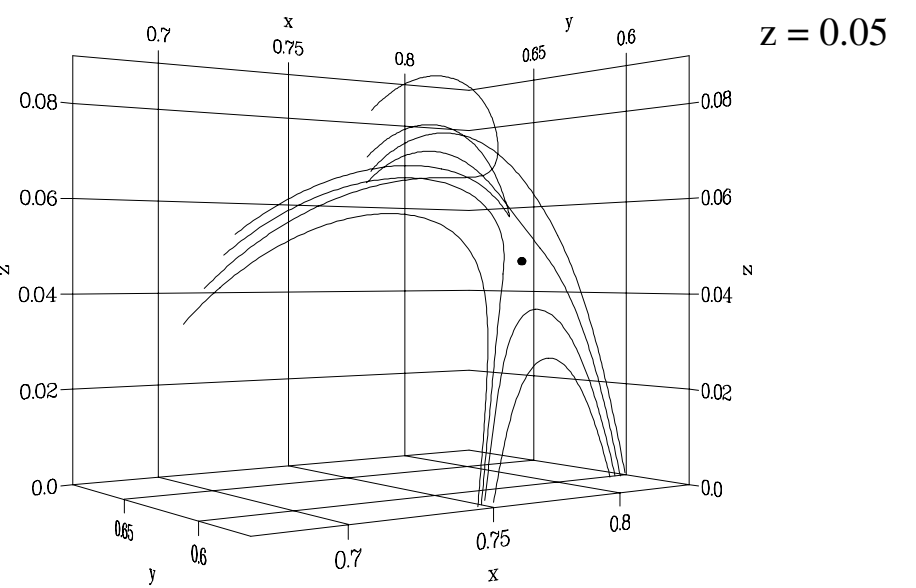

Fig. 11. Fieldlines of $\boldsymbol{c}_{\mathrm{A}}^{(0)}$ around the point $\boldsymbol{x}_{0}=(0.77,0.58,0.05)$, for $\alpha=7 \pi$. The point $x_{0}$ is represented by a dot. The value of the Kolmogorov entropy at that location is $H\left(\boldsymbol{x}_{0}\right)=195.5$.

the perturbation continues to propagate along the fieldline. In the latter case, since all fieldlines start and end at the base $z=0$, the perturbation element will reach the coronal base where it is partially reflected (Paper I). After the reflection, the perturbation element will follow the same fieldline traveling in the opposite direction, so that it will approach the same separator line again. The result is a further strong increase in the wavevector $k$. This process could be repeated a number of times, until $k$ becomes large enough to allow for the dissipation of the perturbation. The amount of dissipated energy also depends on energy losses at each reflection. However, it is clear that those pertubation elements that are more easily dissipated are those propagating along fieldlines that approach the magnetic separators.

Since the parallel thermal conduction is very high in the coronal plasma, even if dissipation takes place mostly close to a separator, the produced heat will rapidly propagate along the magnetic lines. These fieldlines are those traveling close to the separatrix surfaces (see Figs. 10 and 11). The conclusion is that the dissipation of Alfvénic perturbations should preferentially heat the plasma lying around separatrices.

\section{Summary and conclusions}

In this paper we have characterized the property of small-scale generation in small-amplitude Alfvénic perturbations propagating in an inhomogeneous equilibrium magnetic structure, within the framework of a WKB approximation (Petkaki et al. 1998; Malara et al. 2003). The growth of the wavevector $k$ in the perturbation is intimately related to the property that nearby field lines of the $\boldsymbol{c}_{\mathrm{A}}^{(0)}$ field move apart. This aspect has been quantitatively characterized by the Kolmogorov entropy of magnetic lines $H$ (Rechester et al. 1979; Bickerton 1997), which is the rate of separation of nearby lines.

It has been assumed that perturbations have a small amplitude with respect to the equilibrium structure: $\delta v / c_{\mathrm{A}} \ll 1$. It is commonly assumed that in corona the Alfvén velocity is $c_{\mathrm{A}} \sim 10^{3} \mathrm{~km} \mathrm{~s}^{-1}$, while typical values of velocity fluctuation $\delta v \sim 20-40 \mathrm{~km} \mathrm{~s}^{-1}$ can be deduced from measures of nonthermal broadening of coronal lines (Acton at al. 1981; Warren et al. 1997; Chae et al. 1998). Thus, the small-amplitude assumption for fluctuations is verified in the corona. This assumption is commonly used to neglect nonlinear terms in the MHD 
equations, which could give rise to a transfer of the energy of Alfvénic fluctuations to compressive modes. Nakariakov et al. (1997) have shown that phase-mixing of Alfvén waves, and the consequent increase in magnetic pressure gradient, gives rise to magnetosonic waves, even for a small-amplitude wave. A similar effect could also be present in the case considered here, since it is driven essentially by the increase in the wavevector of Alfvén wave. However, provided that the Alfvén wave amplitude is not strongly nonlinear $\left(\delta v / c_{\mathrm{A}} \lesssim 0.1\right)$, the growth of the driven compressive fluctuation saturates at much smaller amplitudes than that of the Alfvén wave (Botha et al. 2000; Tsiklauri et al. 2001). This suggests that the coupling with compressive modes can also be neglected in the case considered here.

The second assumption is that the typical wavelength of fluctuations $\lambda$ is shorter than the spatial scale $L_{0}$ of the equilibrium structure. The initial condition $\lambda \ll L_{0}$ indicates that the wavelengths considered in our model do not represent the main contribution in the spectrum directly produced by photospheric motions. However, due to the coupling with both transverse and longitudinal inhomogeneities at scale $L_{0}$, waves at wavelengths shorter than $L_{0}$ are formed. This has been suggested, for instance, for waves generated by the chromospheric network activity (Axford \& McKenzie 1992). Moreover, there are indications that in closed magnetic structures, Alfvénic perturbations at shorter wavelengths than the loop length are generated by resonance phenomena (Nigro et al. 2004, 2005) inside closed structures. The dissipation mechanism studied here can be applied to these waves.

We have studied the Kolmogorov entropy $H$ for a 3D inhomogeneous magnetic structure belonging to a wide class of solutions of the force-free equilibrium equations (Nakagawa \& Raadu 1972), which represents a model for a typical configuration of the coronal magnetic field above a quiet-Sun region. We tried to calculate a local value for $H$, because our aim was to find a relation between $H$ and the topological properties of the field $\boldsymbol{c}_{\mathrm{A}}^{(0)}$. Since the calculation of $H$ requires integration of the Eqs. (22) and (23) along a finite distance $s_{1}$, the value of $H$ obtained in this way is not strictly local. A compromise was obtained by choosing a value of $s_{1}$ that is much lower than the spatial scale of the equilibrium magnetic field. Higher values of $H$ are found at lower altitudes, probably because there the structure of the magnetic field is more complex: high- $\kappa$ harmonics contribute to $\boldsymbol{B}^{(0)}$ only at low altitudes, while they rapidly decay for increasing $z$. The most interesting feature is the presence of locations where $H$ reaches much higher values than the average value. There the generation of small scales proceeds faster than anywhere else. A power-law tail in the distribution $f(H)$ suggests that $H$ somehow tends to diverge to $+\infty$ at these locations. However, we need to be cautious about such a divergence because the method used to calculate $H$ does not give a strictly local value. These high- $H$ locations are more relevant when the current $\boldsymbol{j}^{(0)}$ is nonvanishing. This is consistent with the result in Paper I that the fraction of the initial wave energy dissipated within the corona is higher for $\alpha \neq 0$.

The topology of the field $\boldsymbol{c}_{\mathrm{A}}^{(0)}$ at high- $H$ locations corresponds to a quasi-2D $\mathrm{X}$ point. It can be described in terms of two separatrix surfaces that intersect along a separator line (e.g., Priest \& Titov 1996; Priest 2001): the highest values of the Kolmogorov entropy of magnetic lines $H$ are located around the separator. Then, our model indicates that the heating due to the dissipation of Alfvénic perturbations should be mainly localized around the separatrices of the magnetic structure. This result could be susceptible to verification by observations. Such a localization of the heating becomes stronger with increasing the Reynolds number $S$ because higher values of the wavevector $k$ are required to dissipate the perturbation before it leaves the Corona (Paper I). Then, locations where $k$ grows particularly fast become more important in the energy balance with increasing $S$.

The method we used to calculate $H$ could be applied to other equilibrium magnetic structures; for instance, those that are derived by extrapolation in the corona starting from measurements of the magnetic field in the photosphere. Finally, we note that studies of 3D magnetic reconnection (see Priest 2001, and references therein) also predict dissipation and heating at separatrices, even if the physical mechanism generating such a dissipation is different from what is considered in our model.

Acknowledgements. One of us (F.M.) is grateful to Antonio Pasqua for his collaboration in calculating the Kolmogorov entropy of magnetic lines. This work was partially supported by the European Community within the Research Training Network "Turbulence in Space Plamas, Theory, Observation and Simulation".

\section{References}

Acton, L. W., Culhane, J. L., Wolfson, C. J., et al. 1981, ApJ, 224, L137 Alissandrakis, C. E. 1981, A\&A, 100, 197

Axford, W. I., \& McKenzie, J. F. 1992, in Solar Wind Seven, ed. E. Marsch, \& R. Schwenn (Oxford: Pergamon), 1

Belcher, J. W., \& Davis, L. 1971, J. Geophys. Res., 76, 3534

Bickerton, R. 1997, Plasma Phys. Control. Fusion, 39, 339

Botha, G. J. J., Arber, T. D., Nakariakov, V. M., \& Keenan, F. P. 2000, A\&A, 363,1186

Califano, F., Chiuderi, C., \& Einaudi, G. 1990, ApJ, 365, 757

Califano, F., Chiuderi, C., \& Einaudi, G. 1992, ApJ, 390, 560

Chae, J., Schühle, U., \& Lamaire, P. 1998, ApJ, 505, 957

Davila, J. M. 1987, ApJ, 317, 514

Davila, J. M. 1991, in Mechanisms of Chromospheric and Coronal Heating, ed. P. Ulmschneider, E. Priest, \& R. Rosner (New York: Springer-Verlag), 464 Heyvaerts, J., \& Priest E. R. 1983, A\&A, 117, 220

Hollweg, J. 1987a, ApJ, 312, 880

Hollweg, J. 1987b, ApJ, 320, 875

Kappraff, J. M., \& Tataronis, J. A. 1977, J. Plasma Phys., 18, 209

Lee, E. M., \& Roberts, B. 1986, ApJ, 301, 430

Malara, F., Veltri, P., Chiuderi, C., \& Einaudi, G. 1992, ApJ, 396, 297

Malara, F., Primavera, L., \& Veltri, P. 1996, ApJ, 459, 347

Malara, F., Petkaki, P., \& Veltri, P. 2000, ApJ, 533, 523

Malara, F., De Franceschis, M. F., \& Veltri, P. 2003, A\&A, 412, 529

Malara, F., De Franceschis, M. F., \& Veltri, P. 2005, A\&A, 443, 1033 (Paper I)

Mariska, J. T. 1992, The Solar Transition Region (Cambridge Univerisity Press)

Mok, Y., \& Einaudi, G. 1985, J. Plasma Phys., 33, 199

Nakagawa, Y., \& Raadu, M. A. 1972, Sol. Phys., 25, 127

Nakariakov, V. M., Roberts, B., \& Murawski, K. 1997, Sol. Phys., 175, 93

Nakariakov, V. M., Roberts, B., \& Murawski, K. 1998, A\&A, 332, 795

Nigro, G., Malara, F., Carbone, V., \& Veltri, P. 2004, Phys. Rev. Lett., 92, 194501

Nigro, G., Malara, F., \& Veltri, P. 2005, ApJ, 629, L133

Petkaki, P., Malara, F., \& Veltri, P. 1998, ApJ, 500, 483

Pommois, P., Zimbardo, G., \& Veltri, P. 1998, Phys. Plasmas, 5, 1288

Priest, E. 2001, Earth Planets Space, 53, 483

Priest, E. R., \& Titov, V. S. 1996, Phil. Trans. Roy. Soc., 354, 2951

Rechester, A. B., Rosenbluth, M. N., \& White, R. B. 1979, Phys. Rev. Lett., 42, 1247

Ruderman, M. S., Nakariakov, V. M., \& Roberts, B. 1998, A\&A, 338, 1118

Schrijver, C. J., Title, A. M., van Ballegooijen, A. A., Hagenaar, H. J., \& Shine, R. A. 1997, ApJ, 487, 424

Similon, P. L., \& Sudan, R. N. 1989, ApJ, 336, 442

Steinolfson, R. S. 1985, ApJ, 295, 213

Tsiklauri, D., Arber, T. D., \& Nakariakov, V. M. 2001, A\&A, 379, 1098

Tsiklauri, D., \& Nakariakov, V. M. 2002, A\&A, 393, 321

Tsiklauri, D., Nakariakov, V. M., \& Rowlands, G. 2003, A\&A, 400, 1051

Warren, H. P., Mariska, J. T., Wilhelm, K., \& Lamaire, P. 1997, ApJ, 484, L91

Wolf, A., Swift, J. B., Swinney, H. L., \& Vastano, J. A. 1985, Physica D, 16, 285 Zimbardo, G., Veltri, P., \& Malara, F. 1984, J. Plasma Phys., 32, 141

Zimbardo, G., Veltri, P., Basile, G., \& Principato, S. 1995, Phys. Plasmas, 2, 2653 